\title{
Isolation and characterization of reverse transcriptase fragments of LTR retrotransposons from the genome of Chenopodium quinoa (Amaranthaceae)
}

\author{
Bozena Kolano • Edyta Bednara • \\ Hanna Weiss-Schneeweiss
}

Received: 30 January 2013/Revised: 30 April 2013 / Accepted: 28 May 2013/Published online: 11 June 2013

(C) The Author(s) 2013. This article is published with open access at Springerlink.com

\begin{abstract}
Key message High heterogeneity was observed among conserved domains of reverse transcriptase $(r t)$ isolated from quinoa. Only one Ty1-copia $r t$ was highly amplified. Reverse transcriptase sequences were located predominantly in pericentromeric region of quinoa chromosomes.

Abstract The heterogeneity, genomic abundance, and chromosomal distribution of reverse transcriptase $(r t)$ coding fragments of Ty1-copia and Ty3-gypsy long terminal repeat retrotransposons were analyzed in the Chenopodium quinoa genome. Conserved domains of the $r t$ gene were amplified and characterized using degenerate oligonucleotide primer pairs. Sequence analyses indicated that half of Ty1-copia rt (51\%) and $39 \%$ of Ty3-gypsy rt fragments contained intact reading frames. High heterogeneity among $r t$ sequences was observed for both Ty1copia and Ty3-gypsy rt amplicons, with Ty1-copia more heterogeneous than Ty3-gypsy. Most of the isolated $r t$ fragments were present in quinoa genome in low copy numbers, with only one highly amplified Ty1-copia $r t$ sequence family. The gypsy-like RNase $\mathrm{H}$ fragments coamplified with Ty1-copia-degenerate primers were shown to be highly amplified in the quinoa genome indicating either higher abundance of some gypsy families of which $r t$
\end{abstract}

Communicated by R. Schmidt.

B. Kolano $(\bowtie) \cdot$ E. Bednara

Department of Plant Anatomy and Cytology, University of

Silesia, Jagiellonska 28, 40-032 Katowice, Poland

e-mail: bozena.kolano@us.edu.pl

H. Weiss-Schneeweiss

Department of Systematic and Evolutionary Botany,

University of Vienna, Rennweg 14, Vienna, Austria domains could not be amplified, or independent evolution of this gypsy-region in quinoa. Both Ty1-copia and Ty3gypsy retrotransposons were preferentially located in pericentromeric heterochromatin of quinoa chromosomes. Phylogenetic analyses of newly amplified $r t$ fragments together with well-characterized retrotransposon families from other organisms allowed identification of major lineages of retroelements in the genome of quinoa and provided preliminary insight into their evolutionary dynamics.

Keywords Chenopodium quinoa - In situ hybridization . Reverse transcriptase - Ty1-copia and Ty3-gypsy retrotransposons

\section{Introduction}

Mobile genetic elements are ubiquitous and abundant components of nearly all eukaryotic genomes. They are divided into two main groups: DNA transposons (class I) and retroelements (class II; Flavell et al. 1992; Kumar and Bennetzen 1999; Friesen et al. 2001; Du et al. 2010; Tenaillon et al. 2010). Class II retroelements are particularly abundant in plant genomes. They transpose replicatively to new genome locations via RNA intermediates reversely transcribed into DNA prior to their integration into the host genome (Wicker et al. 2007). Many plant genomes accumulate large amounts of mobile genetic elements mainly in the dispersed repetitive DNA fraction (Hawkins et al. 2006). The copy number of retrotransposons may vary even among closely related plant taxa, e.g., transposable element content in Zea luxurians is 1.35 -fold greater than in Zea mays (Tenaillon et al. 2011). Retrotransposons can further be divided into two groups depending on the presence/absence of long terminal repeats 
(LTRs). Within the LTR retrotransposons, two subclasses, Ty1-copia and the Ty3-gypsy, are particularly abundant and well analyzed in plants. These two types differ in gene order within the pol domain that encodes protease, RNase $\mathrm{H}$, reverse transcriptase $(r t)$, and integrase. The organization of the coding domains in the Ty3-gypsy elements is similar to that of retroviruses (although they often lack putative envelope coding genes), while in the Ty1-copia elements the integrase domain is found upstream of the $r t$ domain (Kumar and Bennetzen 1999; Bennetzen 2000). It is generally accepted that retrotransposons affect genome size, organization, and function (Parisod et al. 2009). Not only can they substantially change genome size because of their ongoing replicative mode of transposition (and usually slower rate of removal), but they can also generate mutations by inserting new copies within or near gene(s), or cause chromosomal rearrangements via illegitimate or unequal recombination (Bennetzen 2000; Hawkins et al. 2006; Parisod et al. 2009).

The $r t$ gene of the retroelements has several conserved domains characteristic for individual retroelement families (Xiong and Eickbush 1990; Peterson-Burch and Voytas 2002). Availability of degenerate oligonucleotide primers complementary to the conserved regions of the $r t$ has allowed amplification of $r t$ fragments and sampling retrotransposon diversity in various plant genomes (Brandes et al. 1997; Friesen et al. 2001). Many phylogenetic comparisons of populations of retrotransposons, particularly Ty1-copia-like elements, have been performed, both within and among related groups of taxa (Brandes et al. 1997; Heslop-Harrison et al. 1997; Pearce et al. 2000; Sant et al. 2000; Friesen et al. 2001; Navarro-Quezada and Schoen 2002; Alix et al. 2005; Park et al. 2007; Ma et al. 2008; Parisod et al. 2012). Ty3-gypsy-like elements have also been analyzed in several plant groups although less extensively than Ty1-copia (Suoniemi et al. 1998; Friesen et al. 2001; Alix et al. 2005; Hill et al. 2005; Park et al. 2007; Ma et al. 2008). Varying patterns of chromosomal distribution of individual retroelement families, ranging from dispersed to localized near-centromeric, were documented in plants using fluorescent in situ hybridization (FISH) (Brandes et al. 1997; Belyayev et al. 2001; Friesen et al. 2001).

Quinoa (Chenopodium quinoa Willd) is one of the most important food crops in the Andean region of South America. Its grain has an excellent balance of carbohydrates, lipids, and proteins with essential amino acid compositions optimal for human nutrition (Popenoe et al. 1989). Chenopodium quinoa is an allotetraploid species with $2 n=4 x=36$ chromosomes and relatively small genome (1.487 pg/1C or $1,453.8 \mathrm{Mb}$; Kolano et al. 2012), which shows disomic inheritance for most qualitative traits (Ward 2000). Although recent studies of the quinoa genome have provided some insight into its origin and composition of its repetitive genome fraction (Mason et al. 2005; Maughan et al. 2006; Kolano et al. 2008, 2011), retrotransposable elements were not analyzed so far.

The present work aims to characterize the diversity of $r t$ fragments of Ty1-copia and Ty3-gypsy retrotransposons from the $C$. quinoa genome amplified and cloned using degenerate primers. It also attempts to examine their heterogeneity, phylogenetic relationships, abundance in the genome, and chromosomal organization.

\section{Materials and methods}

Plant material and isolation of DNA

Seeds of $C$. quinoa cv. Tango were obtained from Dr. Susanne Dobler (Albert-Ludwigs University, Freiburg, Germany). Plants were grown in pots in a greenhouse under a $16 \mathrm{~h}$ photoperiod at $19 \pm 2{ }^{\circ} \mathrm{C}$. Total genomic DNA was extracted from young leaves using the standard CTAB method (Doyle and Doyle 1987).

Polymerase chain reaction (PCR) and cloning of PCR products

The $r t$ domains of copia and gypsy retrotransposons were amplified from the genomic DNA of quinoa using PCR with degenerate primers. The degenerate primer pairs were used to amplify $r t$ domains of Ty1-copia (Flavell et al. 1992) and Ty3-gypsy elements (Friesen et al. 2001). PCR amplification was carried out in GeneAmpPCR System 9700 (Applied Biosystems, USA). The reaction mixture contained $60 \mathrm{ng}$ DNA, $25 \mathrm{pmol}$ of each primer (Genomed, Warsaw, Poland), $0.2 \mathrm{mM}$ of each dNTPs (Genomed), $1 \times$ buffer (including $3.5 \mathrm{mM} \mathrm{MgCl}$ ) and $1 \mathrm{U}$ of GoTag polymerase (Promega, Madison, USA). The PCR program consisted of initial denaturation at $94{ }^{\circ} \mathrm{C}$ for $3 \mathrm{~min}$, followed by 30 cycles at $94{ }^{\circ} \mathrm{C} 1 \mathrm{~min}, 1 \mathrm{~min}$ at $45^{\circ} \mathrm{C}$, and $1 \mathrm{~min}$ at $72^{\circ} \mathrm{C}$, with a final elongation step of $5 \mathrm{~min}$ at $72{ }^{\circ} \mathrm{C}$. PCR products were purified from the gel using the Qiaquick Gel Extraction Kit (Qiagen, Germany) and cloned into pGEM-T Easy vector (Promega) following the manufacturer's instructions. Randomly chosen recombinant colonies were selected for plasmid DNA isolation using a standard mini-prep method (Sambrook et al. 1987). Two independent rounds of PCR amplification and cloning were carried out for both copia and gypsy elements. The presence of inserts of desired length was verified by PCR with M13 primers (Park et al. 2007). The cloned fragments were sequenced in both directions using M13 universal primers and BigDye terminator v3.1 Cycle Sequencing Kit technology (Applied Biosystems) in 3730xl DNA Analyzer 
(Applied Biosystems). Clone names are composed of an abbreviated taxon name, an abbreviation for the type of element (ty for copia and gy for gypsy) and the clone serial number. For example, pCquty1 stands for the Ty1-copiatype clone number 1 from $C$. quinoa.

\section{Dot blot and hybridization}

The genomic abundance of selected $r t$ fragments of Ty1copia and Ty3-gypsy retrotransposons was determined using dot blot hybridization. Plasmids containing clones of $r t$ were purified with the QIAprepMiniprep Kit (Qiagen), quantified (NanoDrop, TermoScientific, USA) and adjusted to a final concentration of $20 \mathrm{ng} / \mu \mathrm{l}$. Thirty microlitres of each cloned sequence was denatured at $96{ }^{\circ} \mathrm{C}$ for $10 \mathrm{~min}$, and transferred onto a positively charged nylon membrane (Roche, Switzerland) using the Dot Blot 96 System (Biometra, Germany). DNA was fixed to the membrane by UV treatment, washed with sterile water, and air-dried. Genomic DNA of $C$. quinoa cv Tango labeled with digoxigenin (DIG Nick Translation Kit, Roche) was used as the DNA probe. Hybridization was performed at $40{ }^{\circ} \mathrm{C}$ according to the manufacturer's instructions using the DIG High Prime DNA Labelling and Detection Starter Kit II (Roche). High stringency washes were performed to retain only hybridization signals at the level of high sequence similarity (90\%: $0.1 \times \mathrm{SSC}$ and $0.1 \%$ SDS at $68{ }^{\circ} \mathrm{C}$ ). Hybridization signals were documented and quantified using ChemiDocXRS (BioRad, USA).

Dot blot hybridization was also used for the estimation of copy number of the abundant pCquty 119 clone. The genomic DNA samples and clone pCquty 119 were denatured in $0.4 \mathrm{M}$ $\mathrm{NaOH} / 0.01 \mathrm{M}$ EDTA for $10 \mathrm{~min}$ at $98{ }^{\circ} \mathrm{C}$ and transferred to a positively charged nylon membrane (Roche) using a vacuum blotter. Clone pCquty 119 labeled with digoxigenin was used as the DNA probe. Hybridization was performed using the DIG High Prime DNA Labelling and Detection Starter Kit II (Roche) as described above. Integrated densities used for the calculation of copy number were obtained using ImageJ software (Schneider et al. 2012).

\section{Sequence analysis}

Cloned sequences were checked against GenBank database for their homology to previously characterized plant retroelement lineages. DNA sequences were manually aligned in BioEdit version 7.1.3.0 (Hall 1999) and the alignment was guided by conserved amino acid domains of Ty1-copia and Ty3-gypsy plant retrotransposons (Xiong and Eickbush 1990). Gaps were introduced to retain open reading frames (ORFs), and primer-binding regions were excluded prior to the analyses. Genetic similarities were calculated on both nucleotide and amino acid levels using p-distances in
MEGA4 (Tamura et al. 2007). The translated amino acid sequences of quinoa $r t$ sequences were compared with elements isolated from related genera (Amaranthus, Beta, and Spinacia species) as well as with previously characterized elements representing main evolutionary lineages of plant Ty1-copia retrotransposons (available in GenBank and GrainGenes 2 database; Wicker and Keller 2007; Llorens et al. 2009). Neighbor-joining (NJ) analyses of the nucleotide data sets were conducted using p-distance in MEGA 4. Pairwise deletion of missing data (gaps) was used to compute the distance matrices. Nodal support was accessed via bootstrapping using 1,000 bootstrap (BS) replicates.

Chromosome preparation and fluorescence in situ hybridization

Young leaves of quinoa were pretreated with $2 \mathrm{mM}$ 8-hydroxyquinoline for $4 \mathrm{~h}$ at room temperature, fixed in methanol:glacial acetic acid (3:1) and stored at $-20{ }^{\circ} \mathrm{C}$ until use. Metaphase chromosome spreads were prepared as described earlier (Kolano et al. 2011). The clones containing $r t$ inserts were labeled with digoxigenin-11-dUTP using PCR with universal M13 primers (Hajdera et al. 2003). Fluorescence in situ hybridization was preformed according to the protocols described by Schwarzacher and Heslop-Harrison (2000) and Kolano et al. (2011). Briefly, the hybridization mixture consisting of $100 \mathrm{ng}$ of labeled DNA probe, $50 \%$ formamide, $2 \times$ SSC, $10 \%$ dextran sulfate, $0.1 \%$ SDS, and $0.3 \mu \mathrm{g} / \mu \mathrm{l}$ of blocking DNA was denatured for $10 \mathrm{~min}$ at $85{ }^{\circ} \mathrm{C}$, and applied to chromosome preparations. The slides and hybridization mixture were denatured together at $72{ }^{\circ} \mathrm{C}$ for $5 \mathrm{~min}$ in an in situ Thermal Cycler (ThermoHybaid, Franklin, USA) and allowed to hybridize in a humid chamber at $37^{\circ} \mathrm{C}$ for $72 \mathrm{~h}$. Stringent washes (two times in $0.1 \times \mathrm{SSC}$ at $42{ }^{\circ} \mathrm{C}$ ) were followed by detection of digoxigenin using FITC-conjugated primary anti-digoxigenin antibody (Roche). Signal was amplified with FITC-conjugated anti-sheep secondary antibody (Jackson ImmunoResearch, Suffolk, UK). Preparations were mounted in antifade solution Vectashield (Vector Laboratories, Peterborough, UK) containing $2 \mu \mathrm{g} / \mathrm{ml}$ of DAPI (4',6-diamidino-2-phenylindole).

\section{Results}

Isolation and sequence characterization of $r t$ fragments

PCR with degenerate primers designed to amplify conserved domains of the $r t$ gene of Ty1-copia (Flavell et al. 1992) and Ty3-gypsy retrotransposons (Friesen et al. 2001) resulted in fragments of expected length (c. 260 and $420 \mathrm{bp}$, respectively). The first round of PCR and cloning 
Table 1 Analyzed $r t$ sequence length in bp (range/variants more common than $25 \%$, including primer regions), number of clones with intact open reading frames (ORFs) and sequence similarity [given as (minimum) average (maximum)] of $r t$ fragments of Ty1-copia and Ty3-gypsy isolated from the Chenopodium quinoa genome

\begin{tabular}{lrlll}
\hline & Number & $\begin{array}{l}\text { Clones with } \\
\text { intact ORF }\end{array}$ & Length & Similarity \\
\hline Ty1-copia & 72 & 37 & $252-305 / 267(26 \%)$ & (36) $58(100)$ \\
Tork/TAR & 30 & 18 & $252-270 / 267(60 \%)$ & (54) $77(100)$ \\
Subclade A-1 & 20 & 17 & $252-267 / 267(75 \%)$ & $(89) 96(100)$ \\
Subclade A-2 & 4 & 0 & $265-267 / 266(50 \%)$ & $(85) 90(97)$ \\
Tork/Angela & 2 & 2 & 264 & 100 \\
Oryco/Ivana & 1 & 1 & 261 & - \\
Retrofit/Ale & 39 & 15 & $254-306 / 261 \& 264(51 \%)$ & $(50) 57(100)$ \\
Subclade B-1 & 4 & 4 & 264 & $(91) 94(98)$ \\
Ty3-gypsy & 75 & 29 & $393-421 / 417(50 \%)$ & $(58) 70(100)$ \\
Del/Tekay & 64 & 28 & $400-421 / 417(55 \%)$ & $(60) 74(100)$ \\
Subclade A-1 & 10 & 5 & $415-417 / 417(50 \%)$ & $(89) 93(98)$ \\
Subclade A-2 & 4 & 2 & $416-417 / 417(50 \%)$ & $(88) 91(94)$ \\
Subclade A-3 & 6 & 2 & $416-421 / 417(67 \%)$ & $(82) 92(95)$ \\
Subclade A-4 & 9 & 6 & $415-417 / 417(89 \%)$ & $(89) 93(100)$ \\
Subclade A-5 & 8 & 4 & $406-417 / 417(62 \%)$ & $(86) 92(98)$ \\
Subclade A-6 & 6 & 3 & $414-417 / 417(67 \%)$ & $(97) 87(84)$ \\
Subclade A-7 & 5 & 1 & $413-417 / 417(40 \%)$ & $(80) 86(92)$ \\
Subclade A-8 & 13 & 5 & $401-419 / 417(38 \%)$ & $(78) 83(96)$ \\
Reina & 11 & 1 & $393-413 / 416(27 \%)$ & $(60) 68(100)$ \\
Subclade B-1 & 7 & 1 & $416-417 / 416(43 \%)$ & $(69) 77(100)$ \\
\hline
\end{tabular}

yielded 39 sequences of Ty1-copia rt and 43 sequences of Ty3-gypsy $r t$ and the second independent round of PCR and cloning yielded 33 Ty1-copia rt clones and 32 Ty3gypsy $r t$ clones. In total, 72 clones of Ty1-copia $r t$ and 75 clones of Ty3-gypsy $r t$ with homology to known retroelements (GenBank) were selected for further analysis. The sequences are deposited in GenBank under accession numbers: JN575483-JN575554 (Ty1-copia) and JN594743JN594817 (Ty3-gypsy).

Isolated Ty1-copia $r t$ fragments ranged from 252 (pCquty86, pCquty46, pCquty37) to 305 bp (pCquty122) in length, most fragments being $267 \mathrm{bp}(26 \%)$ long (Table 1). The Ty3-gypsy $r t$ sequenced ranged from $393 \mathrm{bp}$ (pCqugy71) to $421 \mathrm{bp}$ (pCqugy51) in length, with most abundant variants of 416-417 bp (70 \%; Table 1). The two shortest clones, pCqugy71 (393 bp) and pCqugy126 (396 bp), lacked primer-binding sites encoding peptide YAKLSKC. Both Ty1-copia and Ty3-gypsy $r t$ sequences were AT-rich with average of $60 \%$ AT content. The putative $r t$ sequences were translated into amino acids and alignment corrected for frame shifts were necessary to maintain an ORF. Thirty-five out of 72 Ty1-copia $r t$ sequences (49\%) contained premature stop codons and/or indels disrupting the reading frame, while the remaining 37 sequence fragments $(51 \%)$ were potentially functional. Among the Ty3-gypsy $r t$ sequences, 29 clones (39\%) possessed intact reading frames while 46 clones $(61 \%)$ had disrupted reading frames. Alignment of putative amino acid sequences of the clones revealed some variation in their translated primer sequences, reflecting the heterogeneous nature of these sequences in the quinoa genome, which may impact the pool of fragments amenable for amplification with degenerate primers.

High nucleotide heterogeneity was observed among isolated $r t$ fragments. The average sequence diversity among Ty1-copia $r t$ elements reached $42 \%$, with the maximum diversity of $64 \%$ (Table 1). Only four pairs of identical sequences (pCquty46 and pCquty37; pCquty85 and pCquty82; pCquty104 and pCquty 105; pCquty119 and pCquty118) as well as a group of three identical sequences (pCquty102, pCquty108, and pCquty125) were found. The Ty3-gypsy $r t$ fragments revealed lower sequence heterogeneity compared to Ty1-copia, as evidenced by both significantly lower maximum (42\%) and average nucleotide diversity (30\%; Table 1). Only two pairs of identical gypsy $r t$ sequences were found (pCqugy 46 and pCqugy49; pCqugy84 and pCqugy120). Analyses of the amino acid heterogeneity of the amplified $r t$ fragments also revealed higher diversity among Ty1-copia $r t$ fragments than among gypsy (Ty1-copia: $\max 63 \%$, average $45 \%$; Ty3-gypsy: max $47 \%$, average $26 \%$ ).

Phylogenetic analysis of quinoa Ty1-copia rt clones

Newly amplified sequences of quinoa copia-type $r t$ clones were aligned with $r t$ fragments reported from related 
genera (Amaranthus and Beta; GenBank; Fig. 2) as well as with previously characterized elements representing main recognized evolutionary lineages of plant Ty1-copia retrotransposons (available in GenBank and GrainGenes 2 database; Wicker and Keller 2007; Llorens et al. 2009). The results of phylogenetic analyses allowed identification of major lineages of Ty1-copia amplified from quinoa genome. Comparison of the amino acid sequence of $r t$ of quinoa elements with other Ty1-copia retrotransposons revealed that genome of quinoa harbors at least four major copia lineages described by Wicker and Keller (2007; Fig. 1). The most numerous group (39 elements) of quinoa $r t$ fragments represents a Retrofit/Ale lineage. Quinoa $r t$ fragments from this lineage showed average sequence similarity of $57 \%$ (Table 1). In Retrofit/Ale lineage, only one small subclade (B-1), containing four quinoa sequences with relatively high sequence similarity (91-98 \%) (Table 1; Fig. 1), was recovered, while the remaining sequences were more heterogeneous. Lineage Tork/TAR comprised 30 sequences with average sequence similarity of $77 \%$ (Table 1; Fig. 1). Within this lineage, 20 quinoa sequences (subclade A-1) shared similarity of 89-100\% and most of these (17 clones) possessed intact reading frames. The second subclade (A-2) consisted of four quinoa elements (sequence similarity of 85-97\%) all with disrupted reading frames (Table 1). Two quinoa $r t$ fragments belonged to the Tork/Angela lineage and only one quinoa clone (pCquty17) exhibited similarity to Oryco/ Ivana retrotransposons (Table 1; Fig. 1).

The major lineages of copia elements previously amplified from Beta and/or Amaranthus species (Fig. 1) were also found in quinoa genome. Sequences from subclade A-1 share 83-86 \% similarity to Amaranthus sequence AF232978.1. Other sequences from the A-2 clade showed $77-85 \%$ similarity to two Amaranthus elements (AF232960.1; AF232985.1). Clone pCquty124 exhibited $84 \%$ nucleotide sequence similarity to a corresponding region of SALIRE1 retrotransposon from Beta vulgaris.

Phylogenetic analysis of quinoa Ty3-gypsy $r t$ clones

Newly amplified sequences of quinoa Ty3-gypsy $r t$ fragments were aligned to $r t$ fragments reported from related genera (Spinacia and Beta species; Kumekawa et al. 1999; Gindullis et al. 2001), as well as elements representing main evolutionary lineages of plant Ty3-gypsy retrotransposons (Llorens et al. 2009). The dendrogram based on amino acid sequence alignment allowed assignment of quinoa $r$ fragments to Del/Tekay and Reina of the Chromovirus lineage (Fig. 2). Most of the isolated quinoa elements represented the Del/Tekay lineage. This evolutionary lineage contained 64 quinoa $r t$ fragments $(85 \%)$ with average sequence similarity of $74 \%$ (range $60-100 \%$;
Table 1). Reina was represented by 11 quinoa elements with average sequence similarity of $68 \%$ (range 60-100 \%; Table 1). The phylogenetic tree of Ty3-gypsy $r t$ sequences was more structured than the one based on Ty1copia $r t$ fragments. In Del/Tekay lineage eight subclades (A-1 to A-8), containing quinoa sequences with relatively high average sequence similarity (83-93\%) (Table 1; Fig. 2) were recovered. In Reina lineage only one subclade (B-1), containing seven quinoa sequences with average sequence similarity $77 \%$ (Table 1; Fig. 2) was recovered. Nearly all Ty3-gypsy $r t$ fragments with intact reading frames belonged to Del/Tekay lineage. All quinoa fragments from Reina lineage, with the exception of clone pCqugy66, represented potential pseudogenes (possessed stop codons or frameshifts).

Some of the previously reported Ty3-gypsy $r t$ clones from Beta showed high similarity to quinoa elements (Fig. 2), including sequences AJ278752.1 (B. vulgaris) and AJ278751.1 (Beta procumbens) with 72-80 \% similarity to the quinoa elements from Del/Tekay lineage. Schmidt retrotransposon (B. vulgaris) showed 70-74\% similarity to quinoa elements from part of Del/Tekay lineage.

Relative abundance in the genome and chromosomal localization of copia and gypsy $r t$ fragments

The relative abundance of isolated $r t$ clones in the $C$. quinoa genome was estimated using dot blot hybridization. The dilutions of all isolated clones were used to compare their abundance (signal intensity) in the genomic DNA of C. quinoa. Very strong hybridization signals were observed for only two copia rt clones: pCquty118 and pCquty119 (clones with identical nucleotide sequences; Fig. 3), indicating their high abundance in the quinoa genome. The copy number of these clones in $C$. quinoa genome has been estimated as 2,600-3,000 molecules per haploid genome (1C DNA) (Fig. 4). A less intense hybridization signal was observed for the clone pCquty99. The remaining analyzed Ty1-copia $r t$ clones showed only weak or very weak hybridization signals suggesting their lower copy numbers in the quinoa genome.

Dot blot hybridization of Ty3-gypsy rt clones did not reveal the presence of any strongly amplified clones, with all clones hybridizing only weakly to genomic DNA (Fig. 3). Physical distribution of Ty1-copia retrotransposons in the quinoa genome was analyzed in metaphase chromosomes and interphase nuclei. Clones which showed stronger hybridization signals in dot blot tests were used as probes for FISH [pCquty119 (identical with pCquty118) and pCquty99]. Hybridization with pCquty119 probe reveled clear signals in the chromosomes (Fig. 5a). Both the chromosomal distribution and intensity of the hybridization signals of pCquty119 differed among chromosomes. 
Fig. 1 Phylogenetic relationships of Ty1-copia $r t$ fragments of $C$. quinoa and other plants' genomes based on translated nucleotide sequence analyses. Numbers above branches indicate bootstrap support (BS) above $60 \%$. Scale bar indicates genetic distance. The quinoa $r t$ sequences from Retrofit/Ale lineage are labeled with blue, the quinoa $r t$ fragment from Oryco/Ivana lineage with green, elements from Tork/Angela lineage with pink and quinoa sequences from Tork/TAR with violet. A-1, A-2, and $B-1$ represent of subclades of very similar quinoa $r t$ sequences

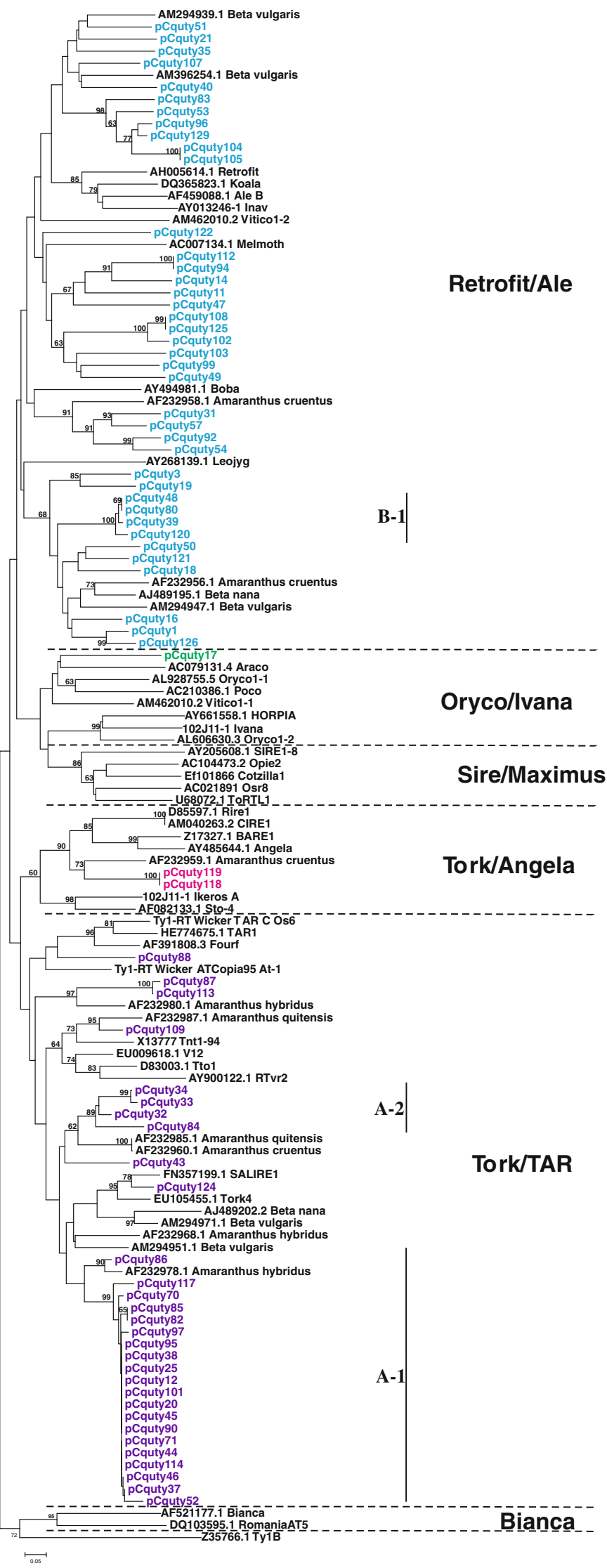


Fig. 2 Phylogenetic relationships of Ty3-gypsy $r t$ fragments amplified from of $C$. quinoa and other plants' genomes based on translated nucleotide sequence analyses. Numbers above branches indicate bootstrap support above $60 \%$. Scale bar indicates genetic distance. The quinoa $r t$ sequences from Del/Tekay lineage are labeled with blue and quinoa elements from Reina lineage with pink, $A-1$ to $A-8$ and $B-1$ indicate subclades of very similar quinoa $r t$ sequences

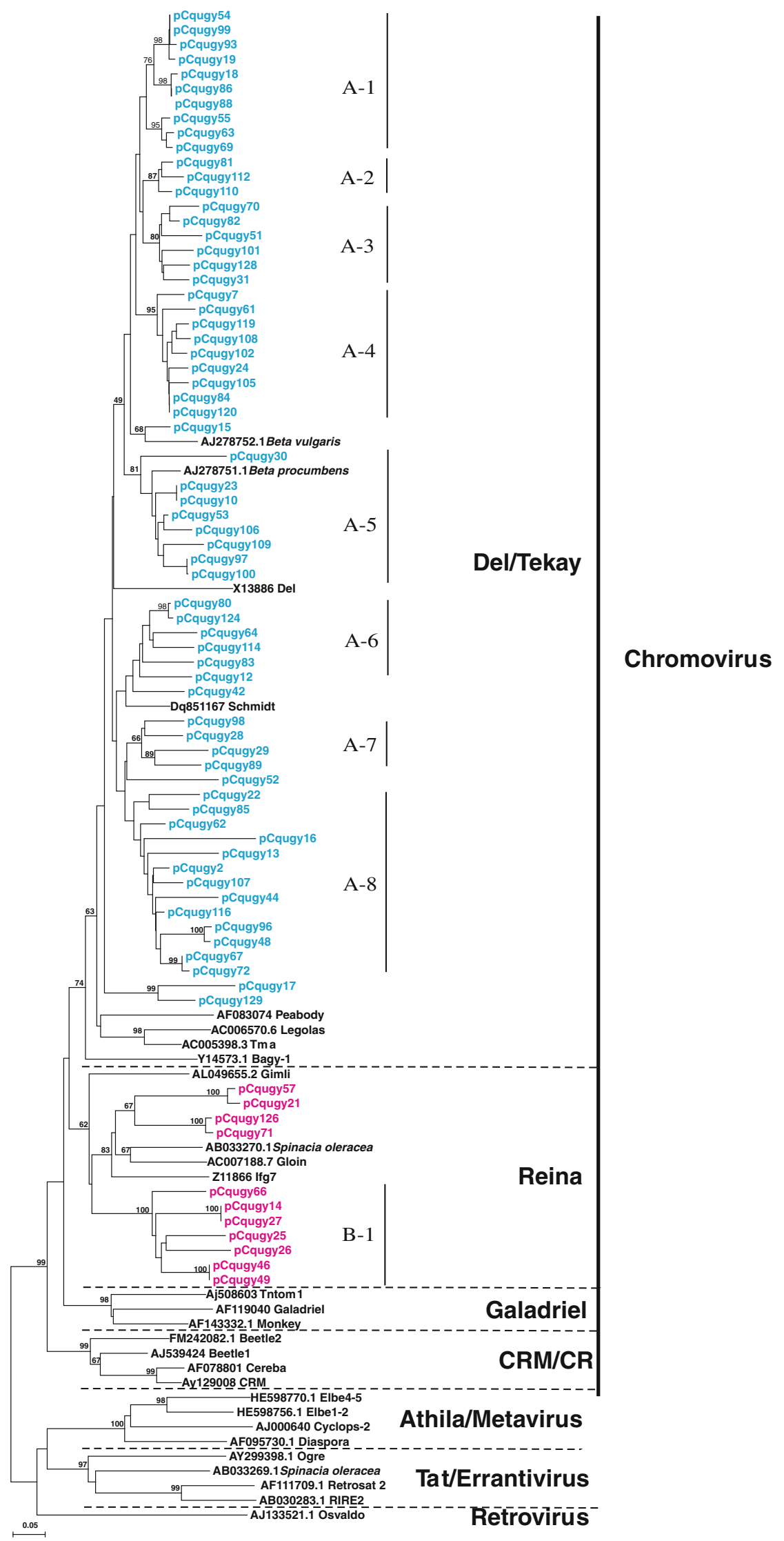




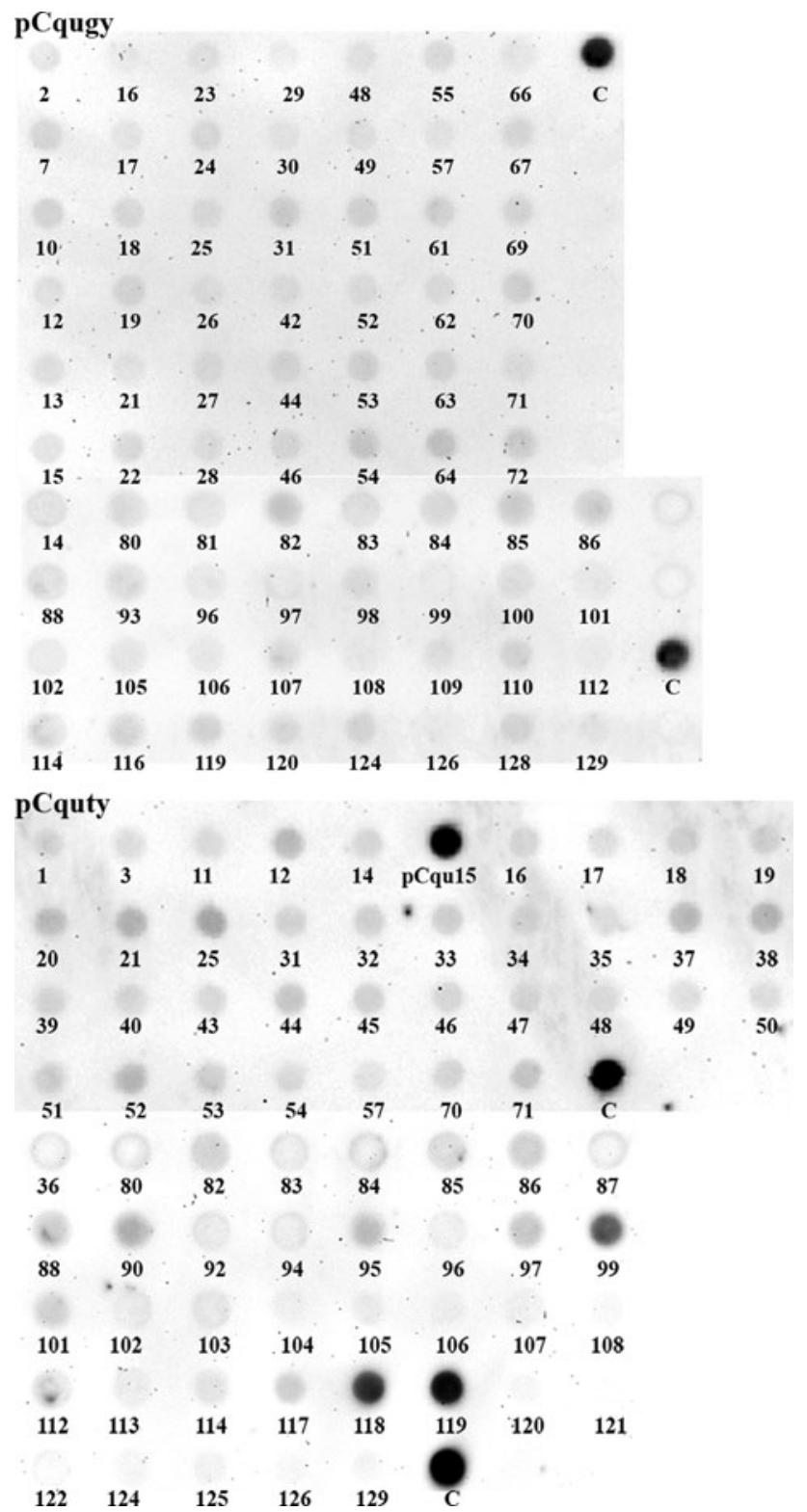

Fig. 3 Relative abundance of Ty1-copia and Ty3-gypsy elements in the $C$. quinoa genome. Dot blot hybridization to Ty1-copia rt and Ty3-gypsy $r t$ clones isolated from $C$. quinoa using genomic DNA of C. quinoa as a probe. Numbers indicate the clone numbers. $C$ positive control (genomic DNA of C. quinoa)

Approximately 16 (out of 36) chromosomes exhibited relatively strong hybridization signals, whereas the rest of the signals were very weak or signals were absent. pCquty119 was predominantly located in pericentromeric regions of the chromosomes and usually absent/undetectable from distal chromosome parts. Scattered hybridization signals were also observed in interphase nuclei, predominantly coinciding with heterochromatin (bright DAPIstained regions). Hybridization with pCquty99 did not yield any detectable signals, indicating that the copy

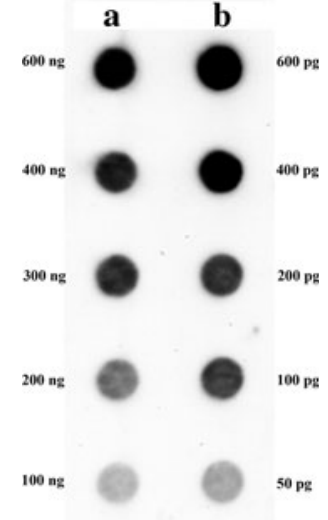

Fig. 4 Dot blot used for the estimation of the copy number of clone pCquty119 in the genome of $C$. quinoa. Different amounts of genomic DNA of $C$. quinoa (row a) and serial dilutions of pCquty119 clone $($ row $b$ ) were dot blotted on a membrane. Labeled clone pCquty119 was used as a DNA probe

number of this sequence is below the detection limit of FISH.

Chromosomal localization of the Ty1-copia elements that appeared to be only weakly amplified in the quinoa genome (dot blot) was tested using the heterogeneous probe cocktail containing all isolated Ty1-copia clones except for the clones pCquty118 and pCquty119. FISH with such a cocktail revealed dispersed hybridization signals in pericentromeric and/or subterminal positions of all chromosomes (Fig. 5b). Hybridization signals observed in interphase nuclei predominantly coincided with heterochromatic regions, but also with some euchromatic regions.

Localization of Ty3-gypsy retrotransposons in interphase nuclei and metaphase chromosomes of $C$. quinoa has been analyzed using the heterogeneous cocktail containing all isolated Ty3-gypsy clones as a probe. All of these clones were only weakly amplified in the quinoa genome (dot blot). FISH signals were observed in most metaphase chromosomes and showed a dispersed pattern with weak clustering, mostly in pericentromeric regions (Fig. 5c). Hybridization signals in the interphase nuclei predominantly co-localized with heterochromatic regions.

Clones pCqu15 and pCqu22: sequence characterization and chromosomal organization

PCR amplification of Ty1-copia $r t$ fragments using standard degenerate primers encoding for TAFLHG and YVDDML resulted in co-amplification of 13 clones with no homology to known rt. Among these, two clones (pCqu22 and pCqu15) were shown to be highly amplified in the $C$. quinoa genome (names of these clones have been 
simplified indicating the species name $C q u$ and serial number only). These two sequences were highly similar (92\%). pCqu22 was 279 bp long, whereas pCqu15 was $2 \mathrm{bp}$ shorter. The alignment of putative amino acid
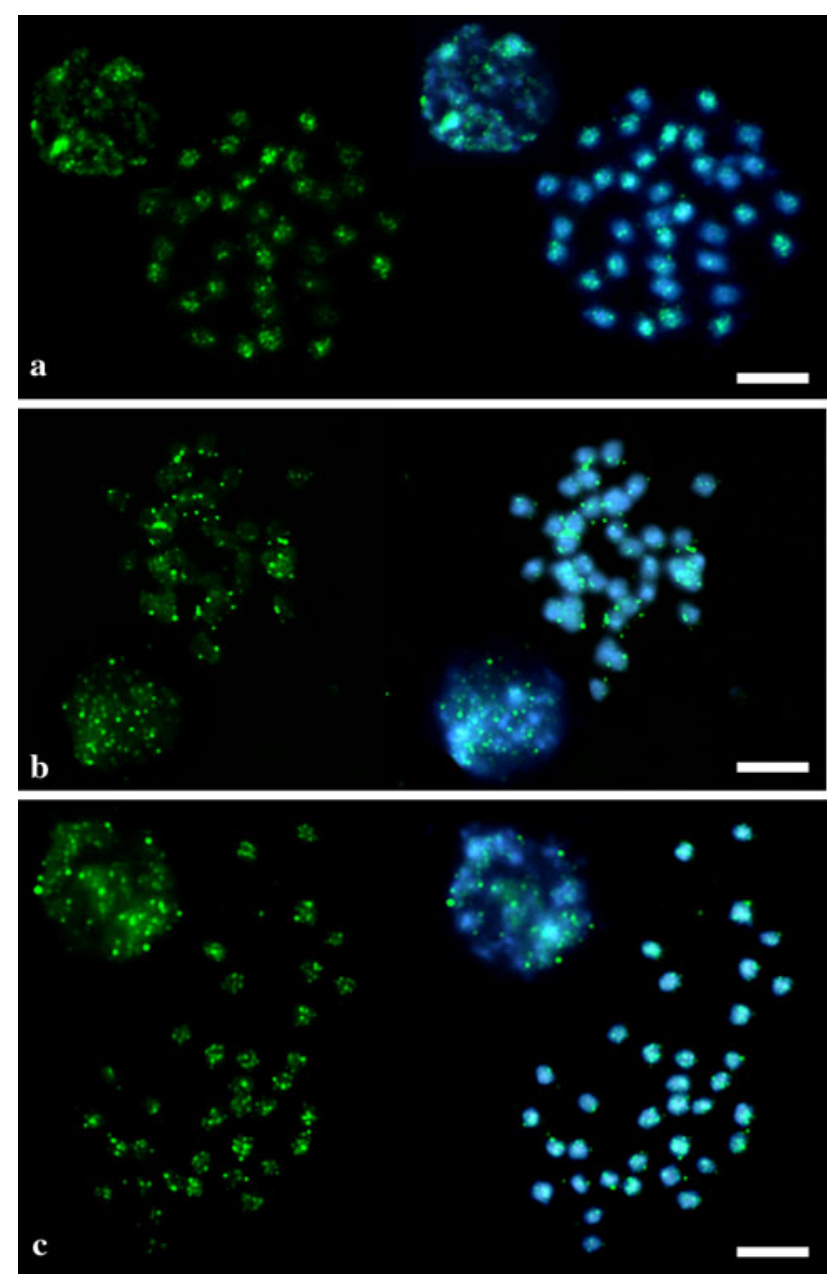

Fig. 5 Fluorescence in situ hybridization of reverse transcriptase fragments (green fluorescence) to DAPI-counterstained metaphase chromosomes and interphase nuclei of C. quinoa: a clone pCquty119; b heterogeneous probe containing all Ty1-copia $r t$ clones except for pCquty118 and pCquty119; c heterogeneous probe representing all Ty3-gypsy $r t$ clones. Scale bar $5 \mu \mathrm{m}$ sequences of these clones revealed presence of a complete upstream primer encoding the TAFLHG domain, whereas deletion of the first three nucleotides has occurred within the downstream primer region (YVDDML). Both sequences showed similarity to a fragment of RNase $\mathrm{H}$ of a Ty3gypsy retrotransposon (blastx against GenBank database; BAE96746.1, AAK52571.1). The sequences are deposited in GenBank under accession numbers: KC869994 and KC869995.

Chromosomal organization of these two sequences was analyzed using FISH with the clone pCqu22. FISH signals were located mainly in the pericentromeric and centromeric regions of all chromosomes, but they varied in intensity. Some chromosomes possessed also faint signals of pCqu22 in more distal regions. This sequence type was absent from nucleolus organizing regions (NOR; Fig. 6). Hybridization signals in interphase nuclei mostly colocalized with DAPI-positive heterochromatic regions. The euchromatic regions of interphase nuclei showed fewer and fainter hybridization signals.

\section{Discussion}

Chenopodium quinoa (Amaranthaceae) is a very important seed crop in South America. Although it is becoming an increasingly popular alternative plant crop also in the USA and Europe, its tetraploid genome origin, structure, and evolution are very poorly understood. Retrotransposons are a source of genetic diversity potentially causing changes in genome structure and gene expression, and are considered to be an important factor in genome plasticity and evolution (Kidwell and Lisch 1997; Ma et al. 2005; Hawkins et al. 2006; Zedek et al. 2010; Lisch 2013). To our knowledge, this study is the first to survey the diversity of LTR retrotransposons in the $C$. quinoa genome. Reverse transcriptase domains of two superfamilies Ty1-copia and Ty3-gypsy were amplified from the $C$. quinoa genome using degenerate primers. PCR amplification of heterogeneous templates typically suffers from bias toward some
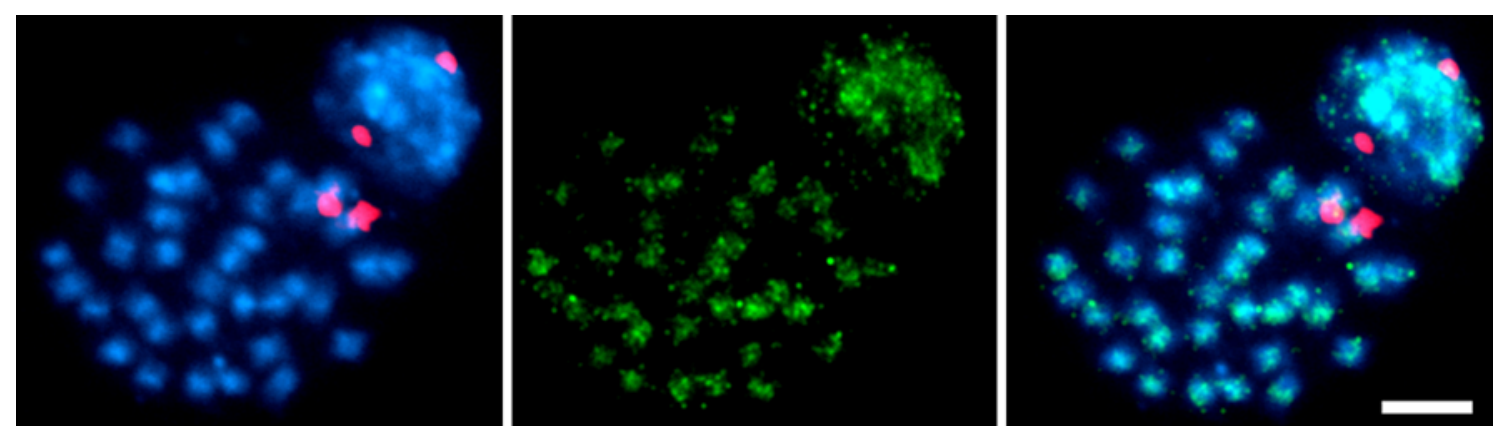

Fig. 6 Fluorescence in situ hybridization of pCqu22 clone (green fluorescence) and 35S rDNA (NOR) (red fluorescence) to DAPIcounterstained metaphase chromosomes and interphase nuclei of C. quinoa. Scale bar $5 \mu \mathrm{m}$ 
sequence types and underrepresentation of others. Such bias is further enhanced by the choice of degenerate primers. In an attempt to at least partly alleviate such methodological effects, two independent rounds of amplification and cloning were conducted and more than 70 clones of each retrotransposon superfamilies (copia and gypsy) were analyzed. Such an approach should have increased chances of obtaining more representative genomic sampling of analyzed retroelements and allow for broader sampling of their diversity in the $C$. quinoa genome. The second PCR round yielded only two new Ty1copia element types, which did not belong to any strongly supported clades and no new Ty3-gypsy $r t$ types. The results suggest that the obtained $r t$ clones reasonably well represent the retroelement diversity in the quinoa genome (Park et al. 2007). One should, however, bear in mind that the degenerate primers were designed based on limited numbers of retrotransposon types and some lineages of retrotransposons might have been underrepresented in the amplification using those primers (Park et al. 2007).

Previous survey of LTR retrotransposons in land plants defined six major common evolutionary Ty1-copia lineages: Tork/TAR, Tork/Angela, Sire/Maximus, Oryco/Ivana and Retrofit/Ale, and Bianca (Wicker and Keller 2007; Llorens et al. 2009). Sire/Maximus and Bianca were the only Ty1-copia lineages not found among newly amplified quinoa $r t$ fragments. Oryco/Ivana, the second lineage of the Sireviruses was represented in our dataset only by one clone. Among Ty3-gypsy elements, typically three major evolutionary lineages are distinguished in land plants (Llorens et al. 2009; Du et al. 2010): non-chromodomain LTR retrotransposons Athila and Tat and Chromovirus lineage. Within the latter several sublineages are commonly described: Del/Tekay, Reina, Galadriel, CRM (Chromoviruses). The majority of isolated quinoa Ty3gypsy $r t$ clones represented Del/Tekay lineage. No elements from the Chromovirus sublineages Galadriel and CRM and no clones similar to Athila or Tat lineages were found. Newly amplified quinoa $r t$ fragments clearly were dominated by one Ty3-gypsy and two Ty1-copia evolutionary lineages. Sireviruses have been shown to proliferate quite extensively in many analyzed plant genomes often representing the majority of Ty1-copia elements of the genome [e.g., $\sim 90 \%$ of Ty1-copia complement, c. $20 \%$ of the genome in maize (Bousios et al. 2012)]. Some retrotransposon lineages [Beetle1 (CRM/CR), Weber and Schmidt 2009; Cotzilla1 (Sirevirus), Weber et al. 2010; Athila, Wollrab et al. 2012], reported as highly amplified in the genome of B. vulgaris, a taxon closely related to Chenopodium, were not represented among amplicons from the $C$. quinoa genome. This might reflect real differences of the presence and abundance of different lineages (Du et al. 2010) in the quinoa genome, caused by their different evolutionary dynamics. Alternatively, it can indicate amplification bias caused by failure to amplify $r t$ fragments that distinctly differ in primer-binding regions from the degenerate primers used. More meaningful comparisons would only be possible when the same methods were used to estimate the abundance of retroelement lineages in the above-mentioned genera.

Ty1-copia $r t$ fragments amplified from the quinoa genome exhibited high levels of heterogeneity (average sequence heterogeneity of $42 \%$ ) in agreement with the data reported for various other angiosperm species: e.g., Phelipanche (Orobanchaceae; Park et al. 2007), or Olea europaea ssp. sativa (Oleaceae; Stergiou et al. 2002). Many of the elements (49\%) were not functional and possessed stop codons and/or frameshifts, and the accumulation of mutations indicated that they might be remnants of more ancient amplifications. Elements resulting from older activity cycles often experience deletions or fragmentation by illegitimate or unequal homologous recombination and are rich in mutations of methylated cytosine to thymine in the $r t$ region (Vitte and Bennetzen 2006). On the other hand, evolutionarily younger transposition events often result in relatively homogeneous groups of retroelements, as most likely evidenced by members of subclade A-1 (Hill et al. 2005). This clade consists of 20 highly similar sequences with average sequence diversity of $4 \%$ and mostly intact reading frames. These data imply that this clade might represent the outcome of a more recent amplification event (Baucom et al. 2009a).

The Ty3-gypsy $r t$ clones of quinoa also exhibited significant levels of sequence heterogeneity, but these were lower than of Ty1-copia $r t$ sequences, with an average diversity of $30 \%$. Higher $r t$ sequence similarity resulted in recovery of one large clade corresponding to Dell/Tekaylike lineage, subdivided into several smaller very similar sequence groups (average similarity range from 83 to $93 \%$ ). These data are consistent with a model of evolutionarily recent amplification of a relatively small subset of closely related ancestral elements. The remaining sequences form Reina lineage most likely represent older retrotransposition events as evidenced from disrupted reading frames and higher nucleotide diversity. Overall lower sequence divergence of Ty3-gypsy $r t$ in comparison to Ty1-copia $r t$ elements was also reported previously in other species groups, e.g., Sorghum or Orobanche (Muthukumar and Bennetzen 2004; Park et al. 2007).

Most of the $r t$ sequences analyzed in this study were present in the quinoa genome in low copy numbers, with the exception of the clones pCquty118, pCquty119 (Tork/ Angela lineage) and pCquty99 (Retrofit/Ale lineage). Similarly, in the genomes of species of the genus Beta closely related to Chenopodium, several abundant Ty1copia [Cotzilla1 (Sire/Maximus), SALIRE1 (Tork/TAR), 
Weber et al. 2010] as well as Ty3-gypsy retroelements were reported [Bongo3 (Del/Tekay), Beetle7 (CRM/CR), Bingo1 (Reina), Weber et al. 2013; Elbe3 (Athila), Wollrab et al. 2012]. The copy number of individual LTR retroelement families in plant genomes can vary greatly from very few copies to thousands of copies (Du et al. 2010). It has been hypothesized that most of the LTR retrotransposon families, regardless of their age, might contain very low numbers of intact elements (repository) as shown for soybean and maize (Baucom et al. 2009b; Du et al. 2010). Conversely, only a few retroelement families that successfully amplified in the genome in the recent past have been represented in genomes in high copy number with mostly intact reading frames (e.g., only $5 \%$ of LTR retrotransposon families identified in the maize genome were very abundant; Baucom et al. 2009b). Different evolutionary trajectories of various lineages of retroelements (cycles of amplification and deactivation/removal) influence dynamics of genome size and genome rearrangements (Vitte and Bennetzen 2006) generally biased toward genome size increase. Polyploidy, particularly allopolyploidy, might change the dynamics of retrotransposon populations of parental genomes and might promote either genome downsizing (often affecting also retrotransposable element populations) or genome size increase (Hawkins et al. 2006; Renny-Byfirld et al. 2011), and might also play a role in shaping retrotransposon population in quinoa.

Ty1-copia elements, and to a smaller extent also Ty3gypsy elements, were preferentially localized in pericentromeric regions of $C$. quinoa chromosomes. These regions are often heterochromatin rich (also in quinoa) and might be preferentially targeted by retroelement insertions. Not only were the most abundant quinoa copia-rt clones pCquty $119 /$ pCquty 118 preferentially located in pericentromeric heterochromatin, but also the overall retrotransposon density was higher in pericentric regions (as evidenced from FISH with pooled $r t$ regions, excluding pCquty 119/ pCquty118) than in subterminal chromosomal regions. Similar pericentromeric localization was reported for two Ty3-gypsy retrotransposons Beetle (CRM/CR lineage) and Bingol (Reina) in B. vulgaris chromosomes (Weber et al. 2013). However, other Ty3-gypsy elements showed different chromosomal organization in Beta. For example, Bongo3 (Del/Tekay lineage) was clustered along all chromosomes with reduced hybridization signal intensity in some of centromeric regions (Weber et al. 2013). SALIRE elements (Ty1-copia, Tork/TAR) isolated from B. vulgaris revealed dispersed hybridization patterns with hybridization signals strongly reduced in the centromeric and pericentromeric regions (Weber et al. 2010). The pericentromeric localization of LTR retrotransposon was reported for several other species, particularly these with small genomes, e.g., Arabidopsis thaliana (Brandes et al.
1997), tomato (Wang et al. 2006), or soybean, where approximately $87 \%$ of the LTR retroelements were found in the recombination-suppressed pericentromeric regions (Schmutz et al. 2010). Selective targeting within heterochromatin might benefit the mobile element by escaping negative selection arising from insertion into genes in distal regions of the chromosome (Gao et al. 2008; Neumann et al. 2011). On the other hand, certain families of retrotransposons commonly found in centromeres of various plant groups were also shown to contribute to centromere function (Neumann et al. 2011). More detailed analyses of composition of retroelement populations in quinoa using next generation sequencing (NGS) should provide more definite answers concerning the potential role of retrotransposons in centromere function.

Two highly repetitive clones bearing similarity to fragment of RNase $\mathrm{H}$ (of Ty3-gypsy retrotransposons), coamplified from the quinoa genome, were localized in chromosomes in a pattern similar to highly amplified $r t$ fragments of the copia type (pCquty119). These sequences might represent another family of retroelements underrepresented in $r t$ amplicons, rearranged elements, or alternatively they might represent a new type of dispersed repeat that originated from retroelements. Current data do not allow inference of the genomic origin and fate of these RNase H-like sequences.

Our study provides the first insight into the composition of the dispersed repetitive DNA fraction of the polyploid quinoa genome and the results of this study contribute the preliminary information important for planning of further studies of retrotransposons in quinoa genome (e.g., isolation of full-length LTR retrotransposons). Both Ty1-copia and Ty3-gypsy retrotransposons recovered from PCR amplification were highly heterogeneous and represented most of the known evolutionary lineages (mainly Tork/TAR and Retrofit/Ale for Ty1-copia; Dell/Tekay and Reina of Chromovirus lineage for Ty3-gypsy). Despite obvious limitations of the method, the combination of PCR amplification, cloning, Southern blotting, and FISH analyses of $r t$ fragments of retrotransposons used in this study provide the first glimpse into diversity and organization of these elements in $C$. quinoa. Recent advances in NGS have began to provide more comprehensive evidence for differential and taxon-specific dynamics of various families of repetitive DNA, including abundant copia and gypsy elements in plants (Macas et al. 2007, 2011; Hribová et al. 2010; Kelly and Leitch 2011). Comparative genomic analyses of putative parental diploids and allopolyploids indicate differential, lineage-specific expansion and removal of various families of retrotransposons during their evolution, resulting in genome size fluctuations (Hawkins et al. 2006; Renny-Byfirld et al. 2011; Parisod et al. 2012). Phylogenetic analyses of whole genus Chenopodium 
implemented with GISH analyses (B. Kolano unpubl.) strongly support allotetraploid origin of $C$. quinoa. Thus, to understand the patterns of its genome evolution, the comparison of the repetitive fraction of quinoa genome with its putative ancestral species will be necessary. Further analyses of the repetitive DNA fraction in $C$. quinoa will be built on data presented in the current study and should provide more detailed insight into organization and evolution of the tetraploid quinoa genome, especially in comparison to diploid progenitors.

Acknowledgments The study was financially supported by the Polish National Science Centre (Project No. N N303 340535 to BK) and the bilateral collaboration program WTZ (Scientific and Technological Cooperation) between Austria (ÖAD) and Poland (Polish Ministry of Science and Higher Education) to BK and HWS (PL 16/2010 and PL 16/2012).

Open Access This article is distributed under the terms of the Creative Commons Attribution License which permits any use, distribution, and reproduction in any medium, provided the original author(s) and the source are credited.

\section{References}

Alix K, Ryder CD, Moore J, King GJ, Heslop-Harrison JS (2005) The genomic organization of retrotransposons in Brassica oleracea. Plant Mol Biol 59:839-851

Baucom RS, Estill JC, Leebens-Mack J, Bennetzen JL (2009a) Natural selection on gene function drives the evolution of LTR retrotransposon families in the rice genome. Genome Res 19:243-254

Baucom RS, Estill JC, Chaparro C, Upshaw N, Jogi A, Deragon JM, Westerman RP, SanMiguel PJ, Bennetzen JL (2009b) Exceptional diversity, non-random distribution, and rapid evolution of retroelements in the B73 maize genome. PLoS Genet 5:e1000732. doi:10.1371/journal.pgen.1000732

Belyayev A, Raskina O, Nevo E (2001) Evolutionary dynamics and chromosomal distribution of repetitive sequences on chromosomes of Aegilops speltoides revealed by genomic in situ hybridization. Heredity $86: 738-742$

Bennetzen JL (2000) Transposable element contributions to plant gene and genome evolution. Plant Mol Biol 42:251-269

Bousios A, Kourmpetis YAI, Pavlidis P, Minga E, Tsaftaris A, Darzentas N (2012) The turbulent life of Sirevirus retrotransposons and the evolution of the maize genome: more than ten thousand elements tell the story. Plant J 69:475-488

Brandes A, Heslop-Harrison JS, Kamm A, Kubis S, Doudrick RL, Schmidt T (1997) Comparative analysis of the chromosomal and genomic organization of Ty1-copia-like retrotransposons in pteridophytes, gymnosperms and angiosperms. Plant Mol Biol 33:11-21

Doyle JJ, Doyle JL (1987) A rapid DNA isolation procedure for small quantities of fresh leaf tissue. Phytochem Bull 19:11-15

Du JC, Tian ZX, Hans CS, Laten HM, Cannon SB, Jackson SA, Shoemaker RC, Ma JX (2010) Evolutionary conservation, diversity and specificity of LTR-retrotransposons in flowering plants: insights from genome-wide analysis and multi-specific comparison. Plant J 63:584-598
Flavell AJ, Dunbar E, Anderson R, Pearce SR, Hartley R, Kumar A (1992) Ty1-copia group retrotransposons are ubiquitous and heterogeneous in higher-plants. Nucleic Acids Res 20:36393644

Friesen N, Brandes A, Heslop-Harrison JS (2001) Diversity, origin, and distribution of retrotransposons (gypsy and copia) in conifers. Mol Biol Evol 18:1176-1188

Gao X, Hou Y, Ebina H, Levin HL, Voytas DF (2008) Chromodomains direct integration of retrotransposons to heterochromatin. Genome Res 18:359-369

Gindullis F, Desel C, Galasso I, Schmidt T (2001) The large-scale organization of the centromeric region in Beta species. Genome Res 11:253-265

Hajdera I, Siwinska D, Hasterok R, Maluszynska J (2003) Molecular cytogenetic analysis of genome structure in Lupinus angustifolius and Lupinus cosentini. Theor Appl Genet 107:988-996

Hall T (1999) BioEdit: a user-friendly biological sequence alignment editor and analysis program for Windows 95/98/NT. Nucleic Acids Symp 42:95-98

Hawkins JS, Kim H, Nason JD, Wing RA, Wendel JF (2006) Differential lineage-specific amplification of transposable elements is responsible for genome size variation in Gossypium. Genome Res 16:1252-1261

Heslop-Harrison JS, Brandes A, Taketa S, Schmidt T, Vershinin AV, Alkhimova EG, Kamm A, Doudrick RL, Schwarzacher T, Katsiotis A, Kubis S, Kumar A, Pearce SR, Flavell AJ, Harrison GE (1997) The chromosomal distributions of Ty1copia group retrotransposable elements in higher plants and their implications for genome evolution. Genetica 100:197204

Hill P, Burford D, Martin DMA, Flavell AJ (2005) Retrotransposon populations of Vicia species with varying genome size. Mol Genet Genomics 273:371-381

Hribová E, Neumann P, Matsumoto T, Roux N, Macas J, Doležel J (2010) Repetitive part of the banana (Musa acuminata) genome investigated by low-depth 454 sequencing. BMC Plant Biol 10:204. doi:10.1186/1471-2229-10-204

Kelly LJ, Leitch IJ (2011) Exploring giant plant genomes with next-generation sequencing technology. Chromosome Res 19:939-953

Kidwell MG, Lisch D (1997) Transposable elements as sources of variation in animals and plants. Proc Natl Acad Sci USA 94:7704-7711

Kolano B, Plucienniczak A, Kwasniewski M, Maluszynska J (2008) Chromosomal localization of a novel repetitive sequence in the Chenopodium quinoa genome. J Appl Genet 49:313-320

Kolano B, Gardunia BW, Michalska M, Bonifacio A, Fairbanks D, Maughan PJ, Coleman CE, Stevens MR, Jellen EN, Maluszynska J (2011) Chromosomal localization of two novel repetitive sequences isolated from the Chenopodium quinoa Willd. genome. Genome 54:710-717

Kolano B, Siwinska D, Pando LG, Szymanowska-Pulka J, Maluszynska J (2012) Genome size variation in Chenopodium quinoa (Chenopodiaceae). Plant Syst Evol 298:251-255

Kumar A, Bennetzen JL (1999) Plant retrotransposons. Annu Rev Genet 33:479-532

Kumekawa N, Ohtsubo E, Ohtsubo H (1999) Identification and phylogenetic analysis of gypsy-type retrotransposons in the plant kingdom. Genes Genet Syst 74:299-307

Lisch D (2013) How important are transposons for plant evolution? Nat Rev Genet 14:49-61

Llorens C, Muñoz-Pomer A, Bernad L, Botella H, Moya A (2009) Network dynamics of eukaryotic LTR retroelements beyond phylogenetic trees. Biol Direct 4:41. doi:10.1186/1745-61504-41 
Ma JX, SanMiguel P, Lai JS, Messing J, Bennetzen JL (2005) DNA rearrangement in orthologous Orp regions of the maize, rice, and sorghum genomes. Genetics 170:1209-1220

Ma Y, Sun HY, Zhao GL, Dai HY, Gao XY, Li H, Zhang ZH (2008) Isolation and characterization of genomic retrotransposon sequences from octoploid strawberry (Fragaria $\times$ ananassa Duch.). Plant Cell Rep 27:499-507

Macas J, Neumann P, Navrátilová A (2007) Repetitive DNA in the pea (Pisum sativum L.) genome: comprehensive characterization using 454 sequencing and comparison to soybean and Medicago truncatula. BMC Genomics 8:427. doi:10.1186/1471-2164-8-427

Macas J, Kejnovský E, Neumann P, Novák P, Koblížková A, Vyskot B (2011) Next generation sequencing-based analysis of repetitive DNA in the model dioecious plant Silene latifolia. PLoS ONE 6:e27335. doi:10.1371/journal.pone.0027335

Mason SL, Stevens MR, Jellen EN, Bonifacio A, Fairbanks DJ, Coleman CE, McCarty RR, Rasmussen AG, Maughan PJ (2005) Development and use of microsatellite markers for germplasm characterization in quinoa (Chenopodium quinoa Willd.). Crop Sci 45:1618-1630

Maughan PJ, Kolano BA, Maluszynska J, Coles ND, Bonifacio A, Rojas J, Coleman CE, Stevens MR, Fairbanks DJ, Parkinson SE, Jellen EN (2006) Molecular and cytological characterization of ribosomal RNA genes in Chenopodium quinoa and Chenopodium berlandieri. Genome 49:825-839

Muthukumar B, Bennetzen JL (2004) Isolation and characterization of genomic and transcribed retrotransposon sequences from sorghum. Mol Genet Genomics 271:308-316

Navarro-Quezada A, Schoen DJ (2002) Sequence evolution and copy number of Ty1-copia retrotransposons in diverse plant genomes. Proc Natl Acad Sci USA 99:268-273

Neumann P, Navrátilová A, Kobližzova A, Kejnovský E, Hřibová E, Hobza R, Widmer A, Doležel J, Macas J (2011) Plant centromeric retrotransposons: a structural and cytogenetic perspective. Mob DNA 2:4. doi:10.1186/1759-8753-2-4

Parisod C, Salmon A, Zerjal T, Tenaillon M, Grandbastien MA, Ainouche M (2009) Rapid structural and epigenetic reorganization near transposable elements in hybrid and allopolyploid genomes in Spartina. New Phytol 184:1003-1015

Parisod C, Mhiri C, Lim KY, Clarkson JJ, Chase MW, Leitch AR, Grandbastien M (2012) Differential dynamics of transposable elements during long-term diploidization of Nicotiana section Repandae (Solanaceae) allopolyploid genomes. PLoS ONE 7:e50352. doi:10.1371/journal.pone.0050352

Park JM, Schneeweiss GM, Weiss-Schneeweiss H (2007) Diversity and evolution of Ty1-copia and Ty3-gypsy retroelements in the non-photo synthetic flowering plants Orobanche and Phelipanche (Orobanchaceae). Gene 387:75-86

Pearce SR, Knox M, Ellis THN, Flavell AJ, Kumar A (2000) Pea Ty1-copia group retrotransposons: transpositional activity and use as markers to study genetic diversity in Pisum. Mol Gen Genet 263:898-907

Peterson-Burch BD, Voytas DF (2002) Genes of the pseudoviridae (Ty1/copia retrotransposons). Mol Biol Evol 19:1832-1845

Popenoe H, King S, Kalinowski L (1989) Lost crops of the Incas. In: Vietmeyer ND (ed) Little-known plants of the Andes with promise for worldwide cultivation. National Academy Press, Washington, pp 139-161

Renny-Byfirld S, Chester M, Kovarik A, Le Comber SC, Grandbastien M, Deloger M, Nichols RA, Macas J, Novak P, Chase MW, Leitch AR (2011) Next generation sequencing reveals genome downsizing in allotetraploid Nicotiana tabacum, predominantly through the elimination of paternally derived repetitive DNAs. Mol Biol Evol 28:2843-2854
Sambrook J, Fritsch E, Maniatis T (eds) (1987) Molecular cloning: a laboratory manual. Cold Spring Harbor, NY

Sant VJ, Sainani MN, Sami-Subbu R, Ranjekar PK, Gupta VS (2000) Ty1-copia retrotransposon-like elements in chickpea genome: their identification, distribution and use for diversity analysis. Gene 257:157-166

Schmutz J, Cannon SB, Schlueter J, Ma JX, Mitros T, Nelson W, Hyten DL, Song QJ, Thelen JJ, Cheng JL, Xu D, Hellsten U, May GD, Yu Y, Sakurai T, Umezawa T, Bhattacharyya MK, Sandhu D, Valliyodan B, Lindquist E, Peto M, Grant D, Shu SQ, Goodstein D, Barry K, Futrell-Griggs M, Abernathy B, Du JC, Tian ZX, Zhu LC, Gill N, Joshi T, Libault M, Sethuraman A, Zhang XC, Shinozaki K, Nguyen HT, Wing RA, Cregan P, Specht J, Grimwood J, Rokhsar D, Stacey G, Shoemaker RC, Jackson SA (2010) Genome sequence of the palaeopolyploid soybean. Nature 463:178-183

Schneider CA, Rasband WS, Eliceiri KW (2012) NIH Image to ImageJ: 25 years of image analysis. Nat Methods 9:671-675

Schwarzacher T, Heslop-Harrison P (2000) Practical in situ hybridization. BIOS Scientific Publishers, Oxford

Stergiou G, Katsiotis A, Hagidimitriou M, Loukas M (2002) Genomic and chromosomal organization of Ty1-copia-like sequences in Olea europaea and evolutionary relationships of Olea retroelements. Theor Appl Genet 104:926-933

Suoniemi A, Tanskanen J, Schulman AH (1998) Gypsy-like retrotransposons are widespread in the plant kingdom. Plant $\mathrm{J}$ 13:699-705

Tamura K, Dudley J, Nei M, Kumar S (2007) MEGA4: molecular evolutionary genetics analysis (MEGA) software version 4.0. Mol Biol Evol 24:1596-1599

Tenaillon MI, Hollister JD, Gaut BS (2010) A triptych of the evolution of plant transposable elements. Trends Plant Sci 15:471-478

Tenaillon MI, Hufford MB, Gaut BS, Ross-Ibarra J (2011) Genome size and transposable element content as determined by highthroughput sequencing in maize and Zea luxurians. Genome Biol Evol 3:219-229

Vitte C, Bennetzen JL (2006) Analysis of retrotransposon structural diversity uncovers properties and propensities in angiosperm genome evolution. Proc Natl Acad Sci USA 103:17638-17643

Wang Y, Tang XM, Cheng ZK, Mucller L, Giovannoni J, Tanksley SD (2006) Euchromatin and pericentromeric heterochromatin: comparative composition in the tomato genome. Genetics 172:2529-2540

Ward SM (2000) Allotetraploid segregation for single-gene morphological characters in quinoa (Chenopodium quinoa Willd.). Euphytica 116:11-16

Weber B, Schmidt T (2009) Nested Ty3-gypsy retrotransposons of a single Beta procumbens centromere contain a putative chromodomain. Chromosome Res 17:379-396

Weber B, Wenke T, Frömmel U, Schmidt T, Heitkam T (2010) The Ty1-copia families SALIRE and Cotzilla populating the Beta vulgaris genome show remarkable differences in abundance, chromosomal distribution, and age. Chromosome Res $18: 247-263$

Weber B, Heitkam T, Holtgräwe D, Weisshaar B, Minoche AE, Dohm JC, Himmelbauer H, Schmidt T (2013) Highly diverse chromoviruses of Beta vulgaris are classified by chromodomains and chromosomal integration. Mob DNA 4:8

Wicker T, Keller B (2007) Genome-wide comparative analysis of copia retrotransposons in Triticeae, rice, and Arabidopsis revealed conserved ancient evolutionary lineages and distinct dynamics of individual copia families. Genome Res 17:1072-1081

Wicker T, Sabot F, Hua-Van A, Bennetzen JL, Capy P, Chalhoub B, Flavell A, Leroy P, Morgante M, Panaud O, Paux E, SanMiguel 
P, Schulman AH (2007) A unified classification system for eukaryotic transposable elements. Nat Rev Genet 8:973-982

Wollrab C, Heitkam T, Holtgrawe D, Weisshaar B, Minoche AE,

Dohm JC, Himmelbauer H, Schmidt T (2012) Evolutionary reshuffling in the Errantivirus lineage Elbe within the Beta vulgaris genome. Plant J 72:636-651
Xiong Y, Eickbush TH (1990) Origin and evolution of retroelements based upon their reverse-transcriptase sequences. EMBO J 9:3353-3362

Zedek F, Šmerda J, Šmarda P, Bureš P (2010) Correlated evolution of LTR retrotransposons and genome size in the genus Eleocharis. BMC Plant Biol 10:265. doi:10.1186/1471-2229-10-265 